Conclusions: Committed pro-inflammatory IL-17A-producing CCR6 +Th memory cells shift towards anti-inflammatory cells with functional regulatory capacities upon exposure to active vitamin $\mathrm{D}$. This process can contribute to restoring the immunological balance and inhibiting synovial inflammation in RA. Disclosure of Interest: None declared

DOI: 10.1136/annrheumdis-2018-eular.6997

\section{THU0049 DEVELOPMENT OF TFH-TH1 LIKE CELLS THROUGH EPIGENETIC MODIFICATION BY STATS FAMILY FACTORS IN PATIENTS WITH SYSTEMIC LUPUS ERYTHEMATOSUS}

$\underline{\text { X. Ma }}{ }^{1,2}$, S. Nakayamada ${ }^{1}$, S. Kubo ${ }^{1}$, K. Sakata ${ }^{1,3}$, K. Yamagata ${ }^{1}$, Y. Tanaka ${ }^{1}$ ${ }^{1}$ First Department of Internal Medicine, School of Medicine, University of Occupational and Environmental Health, Japan, Kitakyushu, Japan; ${ }^{2}$ Department of Pediatrics, The First Hospital of China Medical University, Shenyang, China: ${ }^{3}$ Mitsubishi Tanabe Pharma, Yokohama, Japan

Background: Systemic lupus erythematosus (SLE) is a prototype of autoimmune disease characterised by chronic immune activation and multiple immunologic phenotypes $^{(1)}$. Among several types of immune cells, T follicular helper (Tfh) cells serve important roles in the development and progression of $\mathrm{SLE}^{(2)}$.

Objectives: To assess the characteristics and mechanisms of differentiation of Tfh cells, we probed the phenotype of T helper cells in patients with SLE and underlying epigenetic modifications by cytokine-induced signal transducer and activators of transcription (STAT) family factors.

Methods: Naive $\mathrm{CD} 4^{+} \mathrm{T}$ cells and memory $\mathrm{CD} 4^{+} \mathrm{T}$ cells were isolated and stimulated by various cytokines and T cell receptor (TCR) in vitro. Expression of characteristic markers of Tfh-Th1-cells and phosphorylation of STATs were analysed by flow cytometry and qPCR. Histone modifications were evaluated by chromatin immunoprecipitation. Peripheral blood mononuclear cells from SLE patients and healthy controls were analysed by flow cytometry and productions of cytokines in serum were tested by cytometric bead array.

Results: Differentiation of CXCR5 ${ }^{+} \mathrm{CXCR} 3^{+} \mathrm{Bcl}-6^{+}{ }^{\mathrm{T}}-\mathrm{bet}^{+} \mathrm{IL}-21^{+} \mathrm{IFN}-\gamma^{+}$Tfh-Th1like cells was induced by IL-12. Among STAT family, STAT1 and STAT4 were phosphorylated simultaneously by IL-12 independent of IFN- $\gamma$ and directly bound on Bcl-6 and T-bet gene loci accompanied by suppression of trimethylated histone 3 lysine 27. Compared with healthy controls, responsiveness of activation of STAT1 and STAT4 by IL-12 and proportion of activated Tfh-Th1-like cells were increased in patients with SLE.

Conclusions: Our findings suggest that IL-12-mediated co-activation of STAT1 and STAT4 alter histone modification, resulting in development of Tfh-Th1-like cells that are characteristically expanded in patients with SLE. These findings could be one of underlying pathogenesis of SLE and potentially helpful towards development of cell-specific treatment.

\section{REFERENCES:}

[1] Tsokos GC. Systemic lupus erythematosus. N Engl J Med. 2011;365 (22):2110-21.

[2] Suarez-Fueyo A, et al. T cells in systemic lupus erythematosus. Curr Opin Immunol 2016;43:32-8.

Acknowledgements: The authors thank Ms. N. Sakaguchi for the excellent technical assistance.

Disclosure of Interest: None declared

DOI: 10.1136/annrheumdis-2018-eular.1441

THURSDAY, 14 JUNE 2018

\section{Rheumatoid arthritis - etiology, pathogenesis and animal models}

\section{THU0050 \\ HYPOXIA INDUCES PRODUCTION OF CITRULLINATED PROTEINS IN HUMAN FIBROBLAST-LIKE SYNOVIOCYTES THROUGH REGULATING HIF1A}

R. Yu, C. Li, L. Sun, L. Jian, Z. Ma, J. Zhao, X. Liu. Department of Rheumatology and Immunology, Peking University Third Hospital, Beijing, China

Background: Hypoxia is a prominent microenvironment feature in a range of disorders including cancer, rheumatoid arthritis (RA), atherosclerosis, inflammatory bowel disease (IBD), infection and obesity. Hypoxia promotes biological functions of fibroblast-like synoviocytes via regulating hypoxia-inducible factor $1 \alpha(\mathrm{HIF} 1 \alpha)$. Dysregulated protein citrullination in RA drives the production of antibodies to citrullinated proteins, a highly specific biomarker of RA. However, the mechanisms promoting citrullination in RA are not yet fully elucidated.
Objectives: In the present study, we investigated whether pathophysiological hypoxia as found in the rheumatoid synovium modulates the citrullination in human fibroblast-like synoviocytes (HFLS).

Methods: HFLS were incubated in a hypoxic chamber $\left(5 \% \mathrm{CO}_{2}\right.$ and $1 \% \mathrm{O}_{2}$, balanced with $\mathrm{N}_{2}$ as indicated) or in a normal incubator containing $5 \% \mathrm{CO}_{2}$ and approximately $20 \% \mathrm{O}_{2}$. The realtime quantitative PCR and western blotting were used to detect the expression of peptidylarginine deiminase 2, peptidylarginine deiminase 4 and citrullinated proteins. HFLS were transfected with HIF1 $\alpha$ siRNA to block the HIF1 $\alpha$ pathway.

Results: In the present study, we found that peptidylarginine deiminase 2 (PAD2) and citrullinated proteins were increased in HFLS after exposed to hypoxia. Moreover, knocking down HIF1 $\alpha$ by hif $1 \alpha$ siRNA ameliorated the expression of PAD2 and citrullinated proteins.

Conclusions: Collectively, the present study provide a new mechanism involved in generating citrullinated proteins: hypoxia promote citrullination and PAD production in HFLS. Concurrently, we also proposed a novel hypoxia involved mechanism in RA pathogenesis. The present study deepens our understanding of the role of hypoxia in the pathogenesis of RA and provides potential therapeutic strategy for RA.

Acknowledgements: We thank Jiansheng Huang for providing language help and revising the manuscript.

Disclosure of Interest: None declared

DOI: 10.1136/annrheumdis-2018-eular.6626

\section{THU0051 TNF RECEPTOR 2 PLAYS AN IMMUNOREGULATORY AND ANTI-INFLAMMATORY ROLE IN ARTHRITIS}

W.-Y. Tseng ${ }^{1,2}$, I.-S. Huang ${ }^{1}$, F. Clanchy ${ }^{1}$, K. Mcnamee ${ }^{1}$, F. Mccann ${ }^{1}$, R. O. Williams ${ }^{1}$. ${ }^{1}$ The Kennedy Institute of Rheumatology, University of Oxford, Oxford, UK; ${ }^{2}$ Division of Rheumatology, Allergy and Immunology, Chang Gung Memorial Hospital at Keelung, Keelung, Taiwan, Province of China

Background: Despite the overall success of TNF $\alpha$ inhibitors in rheumatoid arthritis (RA), up to half of patients are classified as either primary or secondary nonresponders ${ }^{1}$. One hypothesis put forward to explain resistance to anti-TNF $\alpha$ therapy is an ascendant effect of dysregulated regulatory T cells and increased Th17 responses following TNF $\alpha$ blockade. Previous studies have demonstrated that TNFR2 is critical for stabilisation and suppressive function of regulatory T cells ${ }^{2,3}$ However, TNFR2 also activates pro-inflammatory signalling cascades and, to date, the net effect of TNFR2 on the pathogenesis of RA remains unclear.

Objectives: In this study we address this question by assessing the progression of collagen-induced arthritis (CIA) in mice deficient for TNFR1 or TNFR2.

Methods: C57BI/6N.Q (H-2 $\left.{ }^{q}\right)$ mice were immunised with bovine type II collagen emulsified in complete Freund's adjuvant. The mice were monitored daily for arthritis and scored clinically from the day of onset of disease. Mice were culled on day 10 after arthritis onset and spleens, lymph nodes, serum and paws were collected for further analysis.

Results: As expected, TNFR $1^{-/-}$mice were found to be largely resistant to arthritis both clinically and histologically (figure 1). In contrast, there was significantly enhanced disease activity at the clinical and histological levels in TNFR2 ${ }^{-1-}$ mice (figure 1) and this was accompanied by increased expression of the pro-inflammatory cytokines, TNF $\alpha$ and IL-6, reduced numbers of regulatory T cells, reduced FoxP3 expression and reduced expression of the immune inhibitory molecules, $\mathrm{PD}-1$ and LAG3, in TNFR2 ${ }^{-1-}$ mice compared to WT mice.

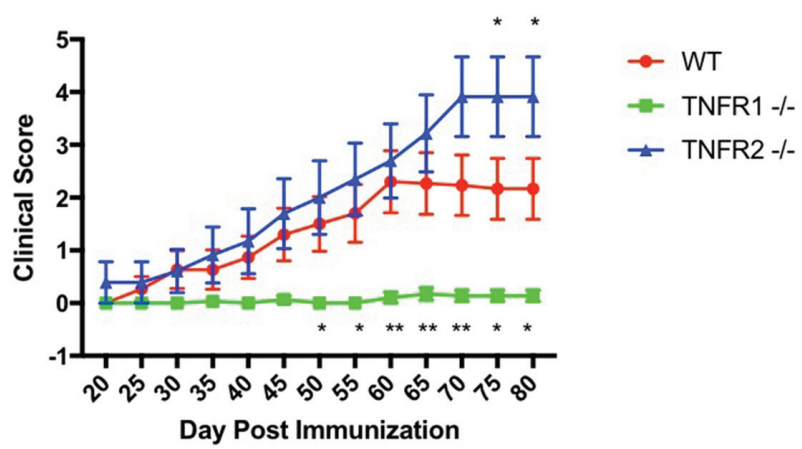

Abstract THU0051 - Figure 1. Progression of CIA in mice deficient for TNFR1 or TNFR2

Conclusions: This study has shown that TNFR2 signalling plays immunoregulatory and anti-inflammatory roles in CIA. First, it contributes to promotion of regulatory T cell generation and FoxP3 expression, and second, it limits the expression of pro inflammatory cytokines. TNFR2 also regulates the expression immune inhibitory 